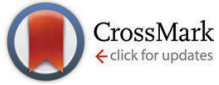

Cite this: J. Mater. Chem. B, 2017, 5, 1005

\title{
Biodegradable nitric oxide precursor-loaded micro- and nanoparticles for the treatment of Staphylococcus aureus biofilms
}

\author{
Sayeed Hasan, ${ }^{a}$ Nicky Thomas, ${ }^{a}$ Benjamin Thierry ${ }^{b}$ and Clive A. Prestidge ${ }^{\star^{a}}$
}

\begin{abstract}
Bacteria in biofilms are more difficult to eradicate than planktonic bacteria and result in treatment challenges for many chronic infectious diseases. Nitric oxide (NO) is an endogenous molecule that offers potential as an alternative to conventional antibiotics; however its sustained topical delivery to biofilms is not readily achieved. With this in mind, we report the development of biodegradable poly(lactide-co-glycolide) (PLGA) based microparticles (MP) and nanoparticles (NP) for encapsulation of the NO precursor isosorbide mononitrate (ISMN) and the controlled delivery to Staphylococcus aureus (S. aureus) biofilms. Firstly, water-in-oil-in-water (w/o/w) emulsification/solvent evaporation methods for PLGA NP and MP syntheses were experimentally optimised with respect to particle size and ISMN loading/encapsulation efficiency. The influence of various experiment parameters, such as the volume of inner aqueous phase, concentration of surfactants, mixing time on the particle size, drug loading and encapsulation efficiency were investigated systematically. Both PLGA MP and NP formulations enabled sustained ISMN release in physiological media over 3 to 5 days. PLGA MP with diameters of $\sim 3 \mu \mathrm{m}$ and ISMN loading of $2.2 \%(\mathrm{w} / \mathrm{w})$ were identified as the optimum delivery system and demonstrated significant antibacterial activity in S. aureus biofilms. This behaviour is considered to be due to targeted biofilm delivery through a combination of effective penetration and sustained release of ISMN.
\end{abstract}

Received 19th December 2016, Accepted 3rd January 2017

DOI: $10.1039 / c 6 t b 03290 \mathrm{~g}$

www.rsc.org/MaterialsB
Nitric oxide (NO) is a potential alternative for antimicrobial therapy to combat both free-floating (planktonic) bacteria and biofilm-associated bacteria as a result of its intrinsic antimicrobial properties. ${ }^{17} \mathrm{NO}$ has been investigated for the treatment of chronic infectious diseases that are frequently associated with bacterial biofilms. ${ }^{15} \mathrm{NO}$ is an endogenously produced gas and has been considered as a promising therapeutic agent in free radical biology investigations due to its remarkable vasorelaxation, antimicrobial and anti-tumour activity. ${ }^{18}$ The clinical application of NO is, however limited due to its short half-life, instability during storage, potential toxicity, lack of efficient localized and systemic delivery and poor dose control. ${ }^{19}$ To overcome these limitations, various NO donors with a range of stabilities have developed to deliver therapeutic levels of NO in biological systems. These NO donors are typically low molecular weight compounds including nitrates, nitrites, N-nitroso, C-nitroso, metal NO complexes and diazeniumdiolates. ${ }^{20}$ The most widely used NO donors are organic nitrates such as glycerol trinitrate (GTN) and isosorbide mononitrate (ISMN), ${ }^{21}$ which have been widely used in the treatment of cardiac diseases. However, the therapeutic potential of NO against biofilms is concentration dependent. Anti-biofilm effects of NO are achieved only at higher concentrations, whereas at suboptimal NO concentrations, S. aureus biofilm growth is enhanced. ${ }^{22}$ The dualistic properties of NO must

\footnotetext{
${ }^{a}$ School of Pharmacy and Medical Sciences, Sansom Institute for Health Research, University of South Australia, Adelaide, South Australia, Australia.

E-mail: clive.prestidge@unisa.edu.au

${ }^{b}$ Future Industries Institute, University of South Australia, Mawson Lakes,

South Australia, Australia
} 
therefore be taken into consideration when designing a topical delivery agent. ${ }^{23}$ To this end, obtaining therapeutic NO levels at an appropriate exposure time is the key challenge towards achieving potent anti-biofilm activity.

To date, there are few reports on the development of particlebased NO delivery systems in which NO donors either encapsulated into or covalently attached to macro- or micromolecular structures and their antibacterial activity in bacterial biofilms. Hetrick et al. synthesized two NONOate derived silica nanoparticle systems, $N$-methylaminopropyltrimethoxysilane/tetraethyl orthosilicate (MAP3/TEOS) and $N$-(6-aminohexyl) aminopropyltrimethoxysilane (AHAP3/TEOS) and reported increased MAP3/ TEOS loading and faster NO release compared to AHAP3/TEOS particles. ${ }^{24}$ The MAP3/TEOS particles exhibited a 1000-fold increase in bactericidal efficacy against $P$. aeruginosa biofilms. Additionally, a significant reduction of planktonic bacteria was reported by using macromolecule NONOate modified silica nanoparticles compared to micromolecule $\mathrm{N}$-diazeniumdiolatemodified proline (PROLI/NO) ${ }^{25}$ Friedman et al. introduced a new silane-based hydrogel nanoparticle platform. The core of this nanoparticle contained nitrite encapsulated within a composite matrix composed of tetramethyl orthosilicate, polyethylene glycol (PEG), chitosan and a glass forming disaccharide. During synthesis, nitrite is thermally reduced to $\mathrm{NO}^{26}$ In a subsequent study, Martinez et al. evaluated the antimicrobial potential of silane based hydrogel nanoparticles. ${ }^{27}$ This study demonstrated that the NO releasing nanoparticles reduced $99.9 \%$ of bacterial growth for cutaneous methicillin-resistant Staphylococcus aureus (MRSA) in a murine infection model. However, the toxicity of silica may limit their potential as a future therapeutic agent.

There is increasing interest in the development of ISMN-based formulations for anti-biofilm applications, owing to its wellestablished efficacy and safety profile. Recently, our group reported on a novel ISMN liposomal formulation for the treatment of S. aureus biofilm-associated chronic rhinosinusitis. ${ }^{23}$ Following the exposure of $S$. aureus biofilm to various liposomal ISMN formulations, multilamellar vesicles (MLV) were found to have greater anti-biofilm effects compared to non-liposomal ISMN and unilamellar vesicle (ULV) liposomes. An increase in the lipid content of liposomal ISMN also improved the anti-biofilm efficacy of both MLV and ULV liposomes. A potential drawback of these systems is the presence of both free ISMN and encapsulated ISMN in the formulation that may cause uncontrolled NO donor delivery to biofilms and potential NO side effects such as hypotension. ${ }^{22}$ Furthermore, liposomal formulations suffer the drawback of poor stability, uncontrolled drug release and low encapsulation efficiency of water-soluble drugs due to rapid drug leakage. The potential use of a successful therapeutic depends on the bioavailability of the therapeutic agents at the site of action. Poly(lactide-co-glycolide) or PLGA micro/nanoparticles have been widely used for encapsulating therapeutic drugs in controlled release applications. Given that PLGA presents inherent advantages over other systems including controlled release rates, biocompatibility/biodegradability, low cytotoxicity and favourable degradation characteristics. ${ }^{28}$ Most importantly, PLGA are a family of FDA-approved biodegradable polymers that have been extensively studied as delivery vehicles for drugs, proteins and peptides. ${ }^{29,30}$ Therefore, PLGA-based formulations are a feasible approach for overcoming the challenges of NO delivery to bacterial biofilms.

Here, we explore the potential of PLGA particles to encapsulate and release ISMN in a controlled manner to kill microbial cells within in vitro $S$. aureus biofilms. We demonstrate that the rate of ISMN release can be tuned by the PLGA particle size and ISMN loading, and quantify anti-biofilm activities of the optimised ISMN-PLGA particulate formulations.

\section{Materials and methods}

\section{Materials}

The biodegradable polymer PLGA (LA/GA ratio 50:50, MW 30 000-60000 Da), polyvinyl alcohol (PVA; 80\% hydrolysed, MW 9000-10 000 Da) and Tween 80 were purchased from Sigma Aldrich, Australia. Isosorbide mononitrate (ISMN) (purity $>95 \%$, MW 191.14) was procured from Bosche Scientific, USA. All other chemical reagents used including acetone, methanol, dichloromethane (Merck, Germany) were of analytical grade. High purity water was obtained from a Milli-Q Direct water purification system (Millipore, USA) and was used throughout the study.

\section{ISMN-loaded PLGA particle preparation}

ISMN-loaded PLGA microparticles (MPs). PLGA MPs containing various amount of ISMN were prepared using a w/o/w double emulsion solvent evaporation technique (Fig. 1). ${ }^{31}$ Briefly, 0.1, 0.5 , and $1 \mathrm{~mL}$ of ISMN aqueous solutions $\left(60 \mathrm{mg} \mathrm{mL}^{-1}\right)$ or pure water (blank PLGA particles) as the internal aqueous phase were emulsified in $10 \mathrm{~mL}$ of dichloromethane containing PLGA (1\%) using a Qsonica Q125 probe sonicator (Newton, CT) for the desired time $(10,30,60$, and $120 \mathrm{~s})$ at $30 \mathrm{~W}$ to obtain a water-in-oil (w/o) emulsion. This primary w/o emulsion was rapidly added into $20 \mathrm{~mL}$ of a $1 \%(\mathrm{w} / \mathrm{v})$ PVA aqueous solution (external aqueous phase) and emulsified again for the same times. The resultant w/o/w double emulsion was then diluted in $150 \mathrm{~mL} 0.3 \%(\mathrm{w} / \mathrm{v})$ PVA aqueous solution in order to minimize droplet coalescence, and was stirred for $4 \mathrm{~h}$ at room temperature to allow evaporation of the organic solvent. The PLGA MPs were collected by centrifugation at $45000 \times g$ (Haake Z36HK, Wehingen, Germany) for $20 \mathrm{~min}$, washed with Milli-Q water three times, and freeze-dried overnight and stored at $4{ }^{\circ} \mathrm{C}$ until further use.

ISMN-loaded PLGA nanoparticles (NPs). PLGA NPs were prepared by specific modification of the w/o/w emulsion technique, ${ }^{32}$ i.e. using high pressure homogenization after the second emulsification step, thereby reducing the size of the dispersed droplets. ${ }^{33}$ Homogenisation was carried out for 3 cycles at 500 bar. The resulting homogenized emulsion was transferred gradually into $150 \mathrm{~mL}$ of a second outer phase containing $0.3 \%(\mathrm{w} / \mathrm{v})$ of PVA stabilizer under continuous stirring to solidify the nanoparticles. The organic solvent was evaporated under magnetic stirring (700 rpm) for 4 hours at room temperature. The generated nanoparticles were collected 
Processing parameters

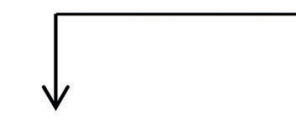

Volume of inner aqueous phase

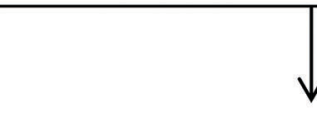

Concentration of surfactants in the external aqueous phase

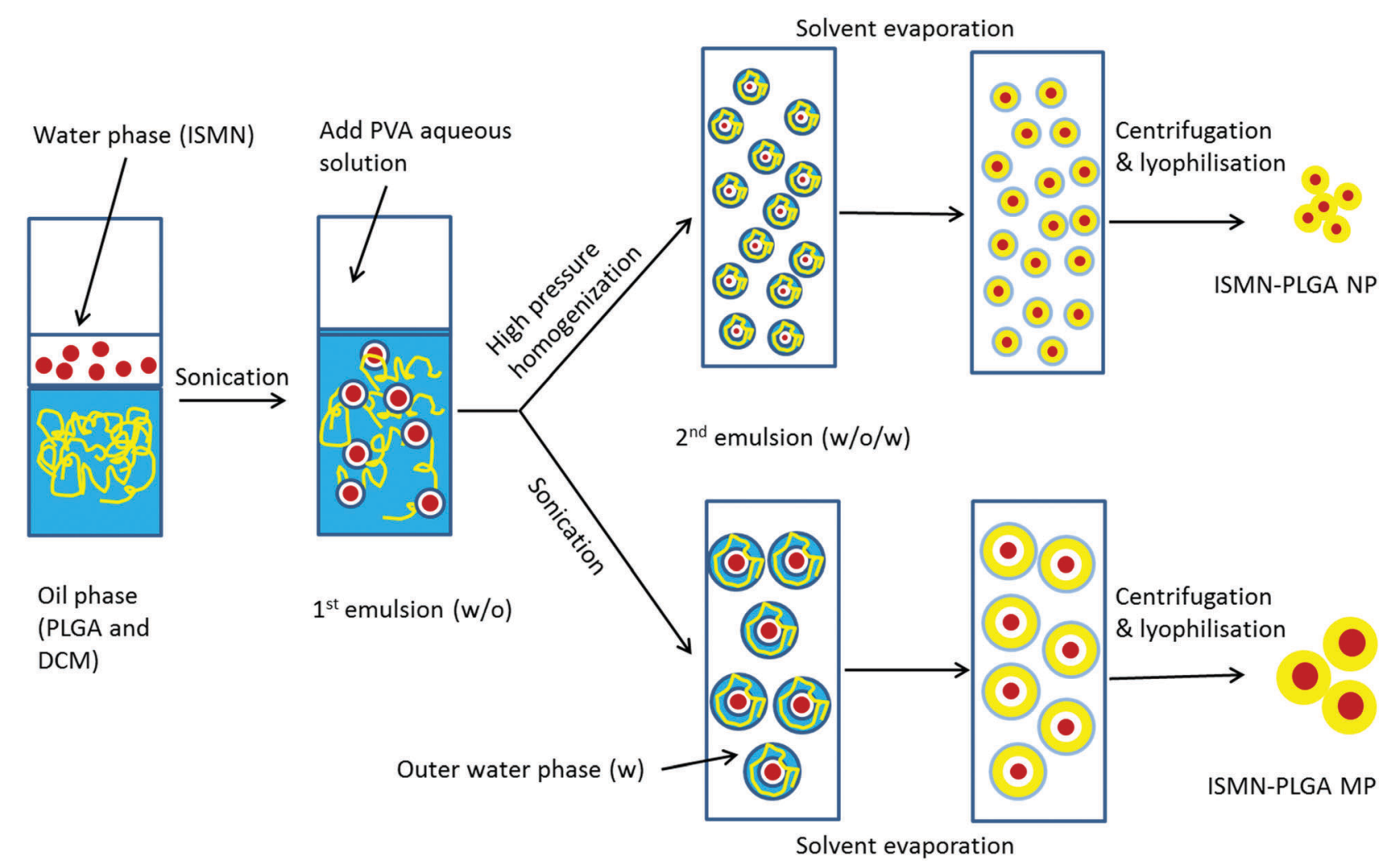

Fig. 1 Schematic of the preparation method of ISMN-loaded PLGA micro- and nanoparticles.

by centrifugation at $45000 \times g$ for $20 \mathrm{~min}$ and washed three times with Milli-Q water. Finally, the products were freeze-dried and stored at $4{ }^{\circ} \mathrm{C}$.

\section{Characterisation of PLGA particles}

Determination of drug loading and encapsulation efficiency. The freeze-dried PLGA MPs and NPs $(10 \mathrm{mg})$ were dissolved in $2 \mathrm{~mL}$ of acetone to release the encapsulated ISMN. Then the mixture was diluted 10 times with HPLC mobile phase ( $80 \%$ Milli-Q water and $20 \%$ methanol), sonicated for $1 \mathrm{~min}$, and centrifuged at $15000 \mathrm{rpm}$ for $10 \mathrm{~min}$. The supernatant was directly injected into a high performance liquid chromatography (HPLC) system (UFLC XR, Shimadzu, Japan). Chromatographic separation was performed on a LiChrospher RP $\mathrm{C}_{18}$ column $(5 \mu \mathrm{m}, 4.6 \mathrm{~mm}$ ID $\times 150 \mathrm{~mm}$, Grace Davidson Discovery Science, Rowville, VIC) at a detection wavelength of $215 \mathrm{~nm}$. The concentration of ISMN was calculated from a standard curve, prepared by measuring the peak areas of known concentrations of ISMN in methanol/water mixtures. Drug loading and encapsulation efficiency were calculated as follows:

$$
\text { Drug loading } \%=\frac{\text { Amount of drug }}{\text { Amount of }(\text { polymer }+ \text { drug })} \times 100
$$

$$
\text { Encapsulation efficiency } \%=\frac{\text { Actual drug loading }}{\text { Theoretical drug loading }} \times 100
$$

Particle size and size distribution. The mean particle diameter size and size distribution of the PLGA-NPs were measured by dynamic light scattering (DLS) using a Zetasizer Nano ZS (Malvern instruments, UK). The analysis was performed by using samples diluted with Milli-Q water. The particle size was expressed as the $z$-average diameter. Values reported are the mean diameter \pm standard deviation for three independent replicate samples.

Scanning electron microscopy. The morphology and particle sizes for both PLGA-MPs and PLGA-NPs were observed using 
scanning electron microscopy (SEM). Lyophilized particles were spread on carbon tape mounted on a SEM aluminum stub and imaged using a Quanta 450 field emission scanning microscope (FEI, Hillsboro, Oregon, USA) at an accelerating voltage of 1-2 kV. For size distribution analysis, the diameters of at least 50 particles were measured and analyzed with Image J software. ${ }^{34}$

Time-of-flight secondary ion mass spectrometry (ToF-SIMS). ToF-SIMS has been used as a highly sensitive surface analytical techniques for characterisation of the ISMN-loaded PLGA microparticle drug delivery system. Specifically, spectral data from a freeze-dried PLGA microparticle powder were acquired to analyse the spatial distribution of the various identified mass fragments of interest. The sample powders were loaded by sprinkling onto adhesive paper, thereby obtaining a finely dispersed powder surface with a low background signal from the substrate. Surface chemical maps were acquired using a PHI TRIFT V nanoTOF instrument (Physical Electronics Inc. Chanhassen, MN, USA) operated with a ${ }^{79+} \mathrm{Au}$ primary ion gun at $30 \mathrm{kV}$ voltage under a vacuum of $5 \times 10^{-6} \mathrm{~Pa}$. 'Unbunched' instrumental settings were applied to optimize spatial resolution for obtaining images from areas roughly $100 \times 100 \mu \mathrm{m}$, with an acquisition time of three minutes. The protonated molecular ion peaks were identified at $m / z=69.03,85.03,127.04$ and $145.04 \mathrm{amu}$ for ISMN and 69.07 amu for PLGA material respectively. Sample spectra and images were analysed with Wincadencen software (Physical Electronics Inc. Chanhassen, MN, USA).

In vitro release studies. The ISMN release kinetics from PLGA MPs and NPs was evaluated in phosphate buffered saline (PBS) at pH 7.4. Briefly, $10 \mathrm{mg}$ of dried ISMN-loaded PLGA MPs (corresponding to $220 \mu \mathrm{g}$ ISMN) and NPs (corresponding to $40 \mu \mathrm{g}$ ISMN) were suspended in $50 \mathrm{~mL}$ of $0.01 \mathrm{M}$ PBS in a flask and incubated in a water bath at $37{ }^{\circ} \mathrm{C}$ with gentle magnetic stirring $(150 \mathrm{rpm})$. At specified time intervals, aliquots $(1 \mathrm{~mL})$ were removed and centrifuged for $20 \mathrm{~min}$ at $25000 \times g$ followed by dilution of the supernatants with mobile phase and ISMN quantification by HPLC as described above. Fresh PBS (1 mL) was added to the particle suspension after each sampling time point and agitation was continued. All release experiments were performed in triplicate.

\section{In vitro bacteria studies}

Bacterial strain and culture conditions. A known biofilmforming $S$. aureus strain, American Type Culture Collection (ATCC) 25923, was used. Briefly, a single loop of S. aureus was propagated from frozen stock, streaked on agar plates (Oxoid Australia) and allowed to grow at $37^{\circ} \mathrm{C}$ for 18 to 24 hours. One or 2 bacterial colonies were suspended in $2 \mathrm{~mL}$ of sterile PBS and adjusted to 1 McFarland standard (equivalent to $3 \times 10^{8}$ colony forming units per $\mathrm{mL}$ ). Then $400 \mu \mathrm{L}$ of this solution was mixed with $5.6 \mathrm{~mL}$ of nutrient broth (1:15 dilution). This suspension served as the inoculum for the formation of biofilms.

Biofilm formation and treatment with ISMN solutions. Sterile 96 well microtiter plates (Sarstedt, Nümbrecht, Germany) were inoculated with $150 \mu \mathrm{L}$ per well of the inoculum and were incubated for 24 hours at $37{ }^{\circ} \mathrm{C}$ with continuously shaking at $70 \mathrm{rpm}$ (Ratek Instruments, Australia) to allow adequate biofilm formation on the well's inner surfaces. After $24 \mathrm{~h}$ incubation the wells containing biofilms were washed twice with PBS to remove adhering planktonic cells. $150 \mu \mathrm{L}$ of nutrient broth containing ISMN with three different concentrations $\left(10,30\right.$ and $\left.60 \mathrm{mg} \mathrm{mL}^{-1}\right)$ were added to the wells and incubated for 1,3 and 24 hours at $37{ }^{\circ}$ C. For quantification of biofilms after 1,3 and $24 \mathrm{~h}$ ISMN exposure, the wells were washed twice with PBS, dried and then stained with $180 \mu \mathrm{L}$ per well of $0.1 \%$ crystal violet (CV) solution. Then plates were incubated for 15-20 min at room temperature. After incubation, the wells containing CV solution were washed with water, dried and then $180 \mu \mathrm{L}$ per well of acetic acid (30\%) was added to solubilize CV for $30 \mathrm{~min} .150 \mu \mathrm{L}$ per well of the solubilized CV solution was then transferred into a new plate and the optical density at $550 \mathrm{~nm}\left(\mathrm{OD}_{550}\right)$ was measured using a plate reader (Model 680, Bio-Rad Laboratories, Hercules, CA, USA). All anti-biofilm assays were carried out in duplicate.

Minimum inhibitory concentration (MIC) studies. The MIC was defined as the lowest concentration of ISMN at which no growth was observed after 24 hours incubation, indicated by an $\mathrm{OD}_{600}<0.1$ measured by a plate reader (Bio-Rad, CA, USA). The MIC value for encapsulated ISMN in PLGA-MPs was determined on planktonic S. aureus (ATCC 25923) using the broth dilution method. ${ }^{35}$ Briefly, $100 \mu \mathrm{L}$ of PLGA-MP suspensions were prepared in nutrient broth using sterile tubes and later inoculated with an equal amount of bacterial suspension to the wells of a microtiter plate to achieve a series of final concentration (3.75-30 $\mathrm{mg} \mathrm{mL}^{-1}$ ) of encapsulated ISMN. The plates were incubated for 24 hours at $37{ }^{\circ} \mathrm{C}$ with continuous shaking at $70 \mathrm{rpm}$. The MIC assays were performed in duplicate.

Minimum biofilm eradication concentration (MBEC) studies. The MBEC was defined as the lowest concentration at which no growth of bacteria from the ISMN-exposed biofilms was observed as indicated by an OD $<0.1$. The MBEC value for encapsulated ISMN in PLGA-MP was determined in S. aureus biofilm using the 96-well plate assay. ${ }^{36}$ Biofilm culture and formation were prepared as previously described. Briefly, the wells containing biofilm were washed twice with PBS to remove adhering planktonic cells after $24 \mathrm{~h}$ incubation, after which $150 \mu \mathrm{L}$ of nutrient broth containing PLGA-MP at a range of 7.5-60 $\mathrm{mg} \mathrm{mL}^{-1}$ ISMN were added to the wells and incubated for another $24 \mathrm{~h}$ at $37^{\circ} \mathrm{C}$. The MBEC assays were performed in duplicate.

Anti-planktonic bacteria studies. In vitro antibacterial studies on planktonic $S$. aureus where undertaken using the Alamar Blue assay. Briefly, $150 \mu \mathrm{L}$ of bacterial suspension was added to tubes containing various amounts of ISMN-PLGA-MP. After $3 \mathrm{~h}$ incubation at $37^{\circ} \mathrm{C}$, a $100 \mu \mathrm{L}$ aliquot was transferred into the wells of a new microtiter plate and added to premixed $100 \mu \mathrm{L}$ nutrient broth and $20 \mu \mathrm{L}$ Alamar Blue dye per well. ${ }^{37}$ The plates were then incubated on a rotating mixer at $10 \mathrm{rpm}$ in the dark at room temperature for 1 hour. Fluorescence of each well was measured $\left(\lambda_{\mathrm{ex}}: 560 \mathrm{~nm}\right.$ and $\lambda_{\mathrm{em}}: 590 \mathrm{~nm}$ ) after $1 \mathrm{~h}$ incubation at $37{ }^{\circ} \mathrm{C}$ by using a microtiter plate fluorometer (Bio-Rad, Australia). ${ }^{38}$ Assays were carried out in duplicate.

Anti-biofilm studies. A biofilm forming $S$. aureus strains (ATCC 25923), was used to test ISMN either as free drug or loaded into PLGA MP using the Alamar Blue assay. S. aureus 
culture and biofilm formation were performed as describe in previous sections. Briefly, the wells containing biofilms were washed twice with PBS to remove adhering planktonic cells after $24 \mathrm{~h}$ incubation. $150 \mu \mathrm{L}$ of nutrient broth containing PLGA-MP at two different ISMN concentration $\left(500 \mathrm{mg} \mathrm{mL}^{-1}\right.$ PLGA-MPs equivalent to $10 \mathrm{mg} \mathrm{mL}^{-1}$ and $2728 \mathrm{mg} \mathrm{mL}^{-1}$ PLGA-MPs equivalent to $60 \mathrm{mg} \mathrm{mL}^{-1}$ ISMN), free ISMN (10 $\mathrm{mg} \mathrm{mL}^{-1}$ and $60 \mathrm{mg} \mathrm{mL}^{-1}$ ISMN) and blank MPs were added to the wells and incubated for another $24 \mathrm{~h}$ at $37{ }^{\circ} \mathrm{C}$. For quantification of biofilms after $24 \mathrm{~h}$ treatments, the wells were washed twice with PBS and then stained with premixed $180 \mu \mathrm{L}$ NB and $20 \mu \mathrm{L}$ Alamar Blue dye per well. Plates were then incubated on a rotating mixer at $10 \mathrm{rpm}$ in the dark at room temperature for 2 hours. Fluorescence ( $\lambda_{\mathrm{ex}}: 560 \mathrm{~nm}$ and $\left.\lambda_{\mathrm{em}}: 590 \mathrm{~nm}\right)$ was measured after $2 \mathrm{~h}$ of incubation $\left(37^{\circ} \mathrm{C}\right)$. Anti-biofilm assays were carried out in duplicate.

Statistical analysis. The statistical analyses of differences in the biofilm biomass resulting from the exposure to PLGA-MP and were tested by one-way analysis of variance (ANOVA) followed by multiple comparison with Bonferroni post hoc evaluation using Graph Pad Prism 5.03 (La Jolla, CA, USA). Differences were considered statistically significant at a level of $p<0.05$.

\section{Results and discussion}

\section{Synthesis and optimization of ISMN loaded PLGA particles}

When considering PLGA particle preparation, the selection of a suitable encapsulation method is largely dependent on the physiochemical properties of the drug. For hydrophilic drug encapsulation, the double emulsion solvent evaporation technique is the most commonly utilized. However, the efficient encapsulation of a low molecular weight and water-soluble molecules such as ISMN into PLGA is a significant challenge. The common double emulsion technique ${ }^{39}$ suffers from poor encapsulation efficiency for water soluble drugs owing to their rapid migration and therefore loss into the aqueous phase. To improve the encapsulation of ISMN into PLGA particles we adjusted specific processing parameters including the volume of the internal aqueous phase, concentration of surfactants and mixing time and evaluated their effect on the particle size, ISMN loading and encapsulation efficiency. ${ }^{40,41}$

Effect of volume of inner aqueous phase. The effect of volume of inner water phase on particle size, ISMN loading and encapsulation efficiency for both PLGA MPs and NPs is reported in Table 1. PLGA-MP and PLGA-NP sizes were significantly affected by the presence of ISMN. Moreover, when the volume of the inner aqueous phase was increased at the same initial ISMN concentration, a decrease in PLGA-MP particle size was observed from $9.8 \mu \mathrm{m}$ to $2.8 \mu \mathrm{m}$. Since the particle size is related to the stability of the initial w/o emulsion, ${ }^{42}$ it is assumed that the optimal volume of the inner aqueous phase may help to stabilize the first emulsion and consequently hampers the fast coalescence of the emulsion droplets. For PLGA-NPs, the observed size increase from $172 \mathrm{~nm}$ to $556 \mathrm{~nm}$ with an increased volume of the inner aqueous phase (Table 1) is considered to have resulted from the less stable w/o emulsion and thus the coalescence of suspended droplets. Meanwhile, the ISMN loading and encapsulation efficiency increased upon increasing the internal aqueous phase volume. For PLGA-MPs, the ISMN loading and encapsulation efficiencies increased when the inner aqueous phase was increased up to $1 \mathrm{~mL}$. The highest ISMN loading $(2.2 \% \mathrm{w} / \mathrm{w})$ and encapsulation efficiency $(23.6 \%)$ for PLGA-MPs were obtained using an inner aqueous phase of $0.5 \mathrm{~mL}$. This can be explained by the localization of ISMN at the interfaces. That is, a larger fraction of ISMN is adsorbed at the outer surface for the lower ISMN concentration and as the concentration is increased, the surface becomes saturated and more ISMN encapsulates in the aqueous core. ${ }^{32}$ If the ISMN amount is further increased, leakage from the inner into the external aqueous phase may occur ${ }^{32}$ hence reducing encapsulation efficiency. Therefore, the ISMN loading and encapsulation efficiency in PLGA-MPs showed a maximum at $0.5 \mathrm{~mL}$ of inner aqueous phase. Interestingly, PLGA-NPs showed no such maximum (Table 1). Considering the delicate balance between the particle size, ISMN loading and encapsulation efficiency, a $0.5 \mathrm{~mL}$ inner aqueous phase was used for all subsequent particle syntheses.

Effect of polyvinyl alcohol (PVA) concentration. The influence of PVA concentration in the external water phase on the PLGA particles is shown in Table 2 (N.B. PLGA and ISMN concentrations and mixing times were kept constant). As the PVA concentration increased from $0.1 \%$ to $1 \%$, the size of PLGA MPs decreased from 3.8 to $2.9 \mu \mathrm{m}$; the ISMN loading level (from $1.7 \%$ to $2.2 \%$ ) and the encapsulation efficiency (from $18.1 \%$ to $23.6 \%$ ) increased. A similar trend was observed for ISMN encapsulation into PLGA-NPs (but a less pronounced influence on size). This behaviour is likely due to enhanced stability of the emulsion droplets at a higher PVA concentration.

Table 1 Effect of volume of inner aqueous phase on ISMN-loaded PLGA particles ${ }^{a}$

\begin{tabular}{|c|c|c|c|c|c|c|}
\hline \multirow[b]{2}{*}{ Volume of internal phase $(\mathrm{mL})$} & \multicolumn{3}{|l|}{ Microparticles } & \multicolumn{3}{|l|}{ Nanoparticles } \\
\hline & Average $\operatorname{size}^{b}(\mu \mathrm{m})$ & DL (\%) & $\mathrm{EE}(\%)$ & Average size (nm) & DL (\%) & $\mathrm{EE}(\%)$ \\
\hline 0.1 & $9.8 \pm 1.8$ & $0.8 \pm 0.28$ & $8.1 \pm 5.0$ & $172 \pm 11$ & $0.14 \pm 0.03$ & $1.6 \pm 0.6$ \\
\hline 0.5 & $2.9 \pm 1.4$ & $2.2 \pm 0.33$ & $23.6 \pm 1.4$ & $264 \pm 21$ & $0.41 \pm 0.02$ & $5.8 \pm 0.1$ \\
\hline 1 & $2.8 \pm 1.7$ & $1.3 \pm 0.20$ & $15.4 \pm 0.7$ & $556 \pm 32$ & $0.49 \pm 0.07$ & $6.3 \pm 0.2$ \\
\hline
\end{tabular}

${ }^{a}$ All batches were emulsified using an ultrasonicator $(30 \mathrm{~W})$ for $60 \mathrm{~s}, 10 \mathrm{~mL}$ PLGA $(1 \%)$ dichloromethane solution was used as organic phase and 1\% PVA (w/v) used as outer aqueous phase. ${ }^{b}$ SEM, At least six representative PLGA MPs were used for the calculation, Data are given as mean $\pm \mathrm{SD}(n=3)$. 
Table 2 Effect of PVA concentration in the external aqueous phase on ISMN-loaded PLGA particles ${ }^{a}$

\begin{tabular}{|c|c|c|c|c|c|c|}
\hline \multirow[b]{2}{*}{ PVA concentration $(\mathrm{w} / \mathrm{v})$} & \multicolumn{3}{|l|}{ Microparticle } & \multicolumn{3}{|c|}{ Nanoparticle } \\
\hline & Average size $(\mu \mathrm{m})$ & DL (\%) & EE $(\%)$ & Size $(\mathrm{nm})$ & DL (\%) & EE $(\%)$ \\
\hline $0.1 \%$ & $3.8 \pm 2.6$ & $1.7 \pm 0.51$ & $18.1 \pm 2.6$ & $271 \pm 17$ & $0.33 \pm 0.62$ & $4.7 \pm 0.3$ \\
\hline
\end{tabular}

${ }^{a}$ All batches were emulsified using $0.5 \mathrm{~mL}$ ISMN aqueous solution (ISMN; $60 \mathrm{mg} \mathrm{mL}{ }^{-1}$ ) was used as internal aqueous phase and $10 \mathrm{~mL}$ PLGA (1\%) dichloromethane solution was used as organic phase.

Effect of ultrasonication time. During the second emulsification step of the PLGA-MP preparation, the intensity and time of emulsification can significantly influence particle size. ${ }^{40}$ The particle size, ISMN loading and encapsulation efficiency as a function of ultra-sonication time are reported in Table 3. Increasing the ultrasonication time up to 60 seconds, decreased particle size, and increased ISMN loading and encapsulation efficiencies; this effect was not so pronounced for longer times. 60 seconds ultrasonication is considered suitable to form well dispersed emulsions, preventing droplet coalescence during the second emulsification step and subsequent ISMN leakage into the outer water phase.

Effect of homogenization pressure. The particle size, ISMN loading and encapsulation efficiency of PLGA NPs were shown to depend on the intensity and extent of homogenization, see Table 4. The particle size decreased from $271 \mathrm{~nm}$ to $101 \mathrm{~nm}$ with increased homogenization pressure and number cycles. However, there was a corresponding reduction in ISMN loading (from $0.41 \%$ to $0.16 \%$ ) and encapsulation efficiency (from $5.8 \%$ to $2.2 \%$ ); this is likely due to ISMN leakage into the outer aqueous phase. 3 cycles at 500 bar pressure were selected to form well dispersed emulsion droplets, a narrow size distribution and to retain ISMN loading.

Based on the above data, the optimal processing parameters were defined as: $0.5 \mathrm{~mL}$ for the inner aqueous phase (ISMN, $60 \mathrm{mg} \mathrm{mL}^{-1}$ ), 1\% PVA concentration (w/v), 1\% PLGA in dichloromethane and either 60 seconds ultra-sonication for MP or 3 cycles of homogenization at 500 bars for NP.

Particle morphology. SEM analysis images of the corresponding PLGA particles are shown in Fig. 2. PLGA-NPs showed evidence of aggregation and hollow structures (Fig. 2A). Conversely, PLGA-MPs displayed more spherical morphologies and homogenous size (Fig. 2B).

Particle surface analysis. The technique of ToF-SIMS provides highly specific surface chemical information to elucidate surface chemistry and can determine the spatial distribution of

Table 3 Effect of ultra-sonication time on ISMN-loaded PLGA-MPs ${ }^{a}$

\begin{tabular}{lllr}
\hline Ultra-sonication time $(\mathrm{s})$ & Average size $(\mu \mathrm{m})$ & DL $(\%)$ & EE $(\%)$ \\
\hline 10 & $5.6 \pm 1.1$ & $0.8 \pm 0.34$ & $8.9 \pm 6.1$ \\
30 & $4.7 \pm 2.5$ & $1.3 \pm 0.29$ & $14.1 \pm 3.6$ \\
60 & $2.9 \pm 1.4$ & $2.2 \pm 0.33$ & $23.6 \pm 1.4$ \\
90 & $3.1 \pm 1.5$ & $2.2 \pm 0.17$ & $24.8 \pm 6$ \\
120 & $3.6 \pm 2.6$ & $2.3 \pm 0.32$ & $25.6 \pm 6.6$
\end{tabular}

${ }^{a}$ All batches were emulsified using an ultrasonicator $(30 \mathrm{~W})$ for $60 \mathrm{~s}$.
Table 4 Effect of homogenization pressure on ISMN-loaded PLGA nanoparticles

\begin{tabular}{lllll}
\hline $\begin{array}{l}\text { Homogenization } \\
\text { pressure (bar) }\end{array}$ & $\begin{array}{l}\text { Number of } \\
\text { cycles }\end{array}$ & Size $(\mathrm{nm})$ & DL (\%) & EE (\%) \\
\hline 500 & 1 & $271 \pm 16$ & $0.40 \pm 0.29$ & $5.6 \pm 0.6$ \\
500 & 3 & $264 \pm 21$ & $0.41 \pm 0.02$ & $5.8 \pm 0.3$ \\
500 & 5 & $243 \pm 43$ & $0.37 \pm 0.21$ & $4.8 \pm 0.8$ \\
1000 & 1 & $139 \pm 62$ & $0.21 \pm 0.02$ & $2.7 \pm 0.2$ \\
1000 & 3 & $121 \pm 31$ & $0.16 \pm 0.04$ & $2.2 \pm 0.1$ \\
1000 & 5 & $101 \pm 34$ & $0.16 \pm 0.08$ & $2.2 \pm 0.4$
\end{tabular}

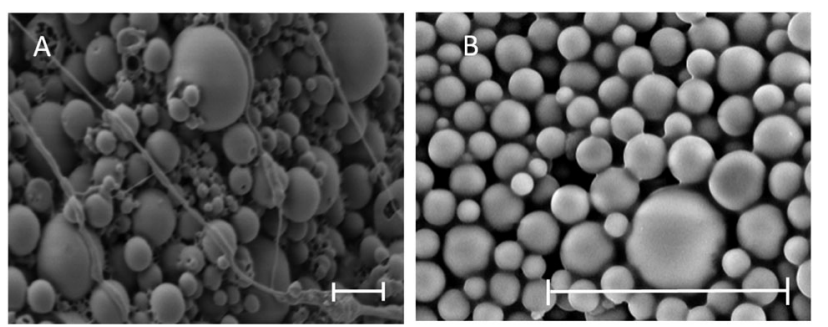

Fig. 2 SEM images of the ISMN-loaded (A) PLGA nanoparticles (scale bar $=300 \mathrm{~nm}$ ) and $(B)$ PLGA microparticles (scale bar $=10 \mu \mathrm{m}$ ).

molecules within a carrier material. ToFSIMS has been previously applied to pharmaceutical microparticle systems. ${ }^{43-45}$ In the current study, we probe the surface chemistry of ISMN containing PLGA microparticles to elucidate whether ISMN is distributed at the particle surfaces or not. Firstly, we determined ToFSIMS spectra of ISMN and PLGA particles to identify the specific mass fragments and the mass fragmentation patterns; these are shown in Fig. 3. No corresponding ISMN fragment peaks was observed except at mass to charge ratio $(\mathrm{m} / \mathrm{z})$ 69.03 amu correspond to ISMN fragment $\mathrm{C}_{4} \mathrm{H}_{5} \mathrm{O}^{+}$and 69.07 amu to PLGA fragment $\mathrm{C}_{5} \mathrm{H}_{9}{ }^{+}$would be overlapping in the spectra. Fig. $4 \mathrm{~A}$ is a total ion image of the PLGA microparticles and Fig. $4 \mathrm{~B}$ is an image associated with ISMN, which also confirm that the ISMN is not located at the particle surface, but fully encapsulated in the sub-surface/bulk of the PLGA particles.

ISMN release in vitro. The release kinetics of ISMN from PLGA micro- and nanoparticles are shown in Fig. 5. It is likely that ISMN release from these carriers is controlled by some combination of diffusion and erosion mechanisms. For PLGANPs, $\sim 50 \%$ of ISMN was released within 1 hour (burst release) and further release was sustained for up to 3 days. For PLGAMPs, $\sim 40 \%$ of the encapsulated ISMN displayed a burst release then, a further sustain release was observed for up to 5 days. 


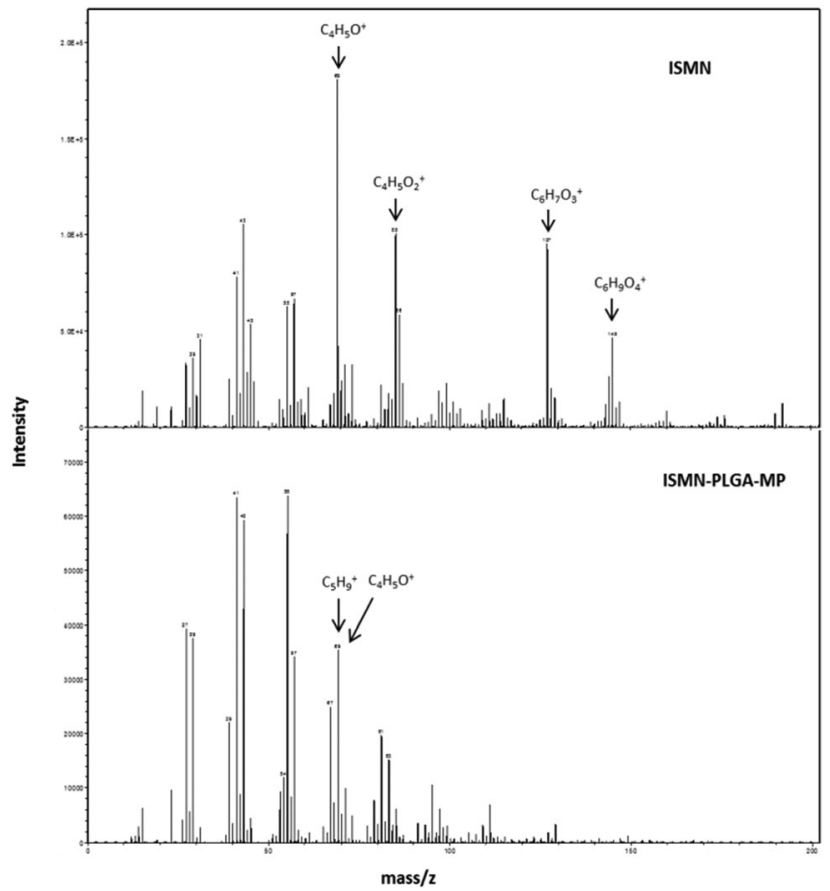

Fig. 3 ToF-SIMS spectra of the surface of ISMN and ISMN-loaded PLGA microparticles from a mass of 0 to 200, peaks used in the subsequent analysis are annotated (the unique ions are used to represent each constituent).

The initial burst release is considered to be due to diffusion controlled mass transport processes, ${ }^{46,47}$ i.e. the small and water soluble ISMN molecules that are encapsulated within the subsurface of the PLGA particles can readily diffuse out of the polymeric particle matrix.

In contrast, the sustained release phase results from several factors such as an increase in the diffusion pathways with time due to polymer swelling, degradation and erosion. ${ }^{47}$

To further elucidate ISMN release mechanisms from these PLGA particles, ISMN release data were fitted to the KorsmeyerPeppas equation as shown in Fig. $6,{ }^{48}$ i.e. $M_{t} / M_{\infty}=k t^{n}$, where $M_{t} / M_{\infty}$ is the fraction of ISMN released at time $t, k$ is the rate constant and $\mathrm{n}$ is the drug release exponent. In this context, $n$ values between 0.45 and 0.89 are indicative of non-Fickian release which refers to a combination of both diffusion and
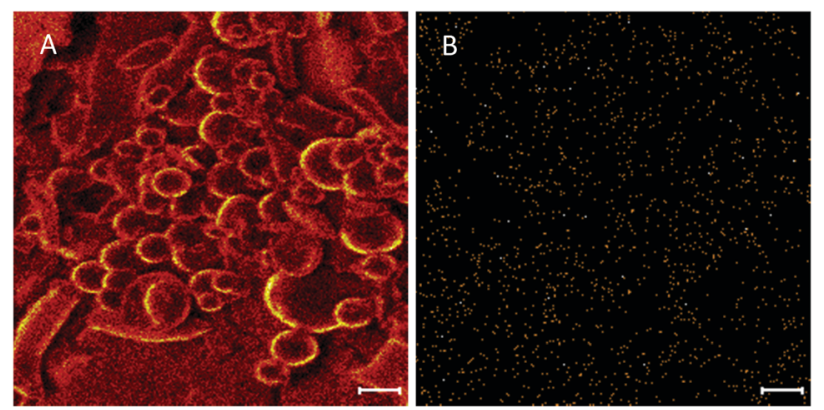

Fig. 4 (A) ToF-SIMS total ion image of PLGA particle and (B) image associated with ISMN (scale bar $=10 \mu \mathrm{m}$ ).

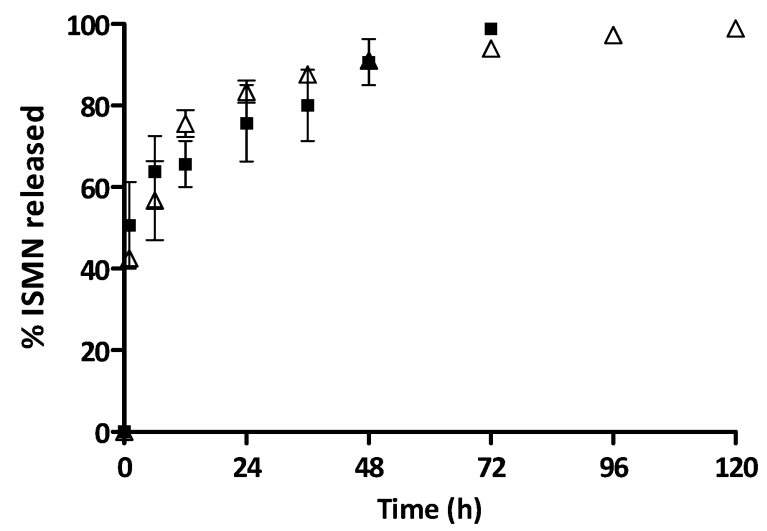

Fig. 5 The percentage of ISMN release from PLGA-MPs $(\triangle)$ and PLGANPs ( $\mathbf{\square})$ at $37{ }^{\circ} \mathrm{C}$ in PBS (pH 7.4), data represent mean $\pm S D, n=3$.

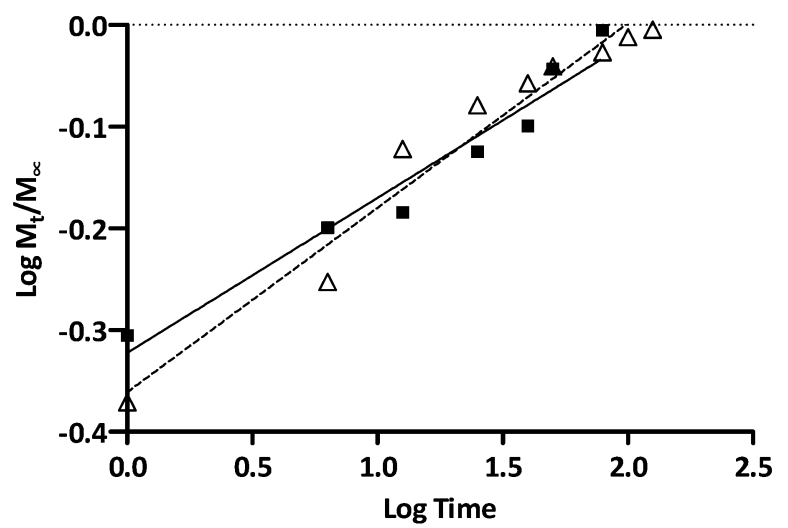

Fig. 6 Korsmeyer and Peppas kinetic plots of ISMN release from PLGA$\operatorname{MPs}(\triangle)$ and PLGA-NPs (

erosion drug release mechanisms. ${ }^{49}$ The observed $\mathrm{n}$ values for the release of ISMN from PLGA-MPs and PLGA-NPs of 0.74 and 0.66 respectively $\left(R^{2}>0.95\right)$ is confirmation of non-Fickian behaviour. Such release characteristics could be attributed to the increase in strong entanglement bonds between the polymer matrixes which resisted the erosion by the dissolution medium in the initial hours of ISMN release. ${ }^{47}$ The drug release from PLGA micro/nanoparticles is a complex process attributed to diffusion followed by degradation and is controlled by the molar ratio of the lactic and glycolic acid in the polymer chain, molecular weight of the polymer, the degree crystallinity and physico-chemical properties of the drug. ${ }^{30}$ Initial release of ISMN from both PLGA micro- and nanoparticles is considered a burst release of deposited or weakly bound ISMN molecules on the sub-surface of micro- and nanoparticles. Jameela et al. ${ }^{50}$ explained that when particles are prepared by the double emulsion method, most hydrophilic drugs exhibit higher tendency to migrate to the aqueous medium, thereby concentrating at the surface or sub-surface of the particles and rapidly released. A potential limiting factor in the preparation of ISMN-containing PLGA micro/nanoparticle is the hydrophobic nature of the PLGA polymer in contrast to the hydrophilic characteristics of ISMN. Generally, encapsulation of hydrophobic drug molecules in 
PLGA preparations is preferred due to strong hydrophobic interactions between the polymer and drug during formulation. Given the high aqueous solubility of ISMN (60 $\left.\mathrm{mg} \mathrm{mL}^{-1}\right)$, it is not surprising that previous reports suggesting its presence on the outer surface or subsurface of particles, forming a molecular layer and thereby susceptible to be easily and rapidly released. ${ }^{51}$ Besides the polymer type, the drug loading also influenced the drug release rate. PLGA micro- and nanoparticle had different ISMN loadings (2.2\% and $0.4 \%$ respectively) and these two ISMN release profiles have similar shape, but the PLGA-MPs with a higher ISMN loading leads to an increased initial rapid release. ${ }^{52}$ After the initial ISMN release from PLGAMPs, there was a sustained ISMN release, indicating the constant degradation of the PLGA and the diffusion of encapsulated ISMN from the inner core of the PLGA-MPs. ${ }^{53}$ In contrast, faster release of ISMN from the PLGA-NPs could result from larger surface area and the shorter diffusion pathways compared to microparticles.

Anti-biofilm effect of ISMN in solution. S. aureus biofilms grown for 1, 3 and 24 hours showed a concentration-dependent response to ISMN solutions, as shown in Fig. 7. ISMN solutions at concentration of 30 and $60 \mathrm{mg} \mathrm{mL}^{-1}$ showed a statistically significance anti-biofilm effect $(P<0.05)$ compared to the untreated control. This effect increased as the time of exposure increased from 1 to 3 to 24 hour. At an ISMN concentration of $10 \mathrm{mg} \mathrm{mL}^{-1}$ a less pronounced anti-biofilm effect was observed for up to 3 hours and then enhanced biofilm growth was observed at 24 hours of exposure. A stimulatory effect of biofilm growth by ISMN at low concentrations (i.e. $3.75 \mathrm{mg} \mathrm{mL}^{-1}$ ) has also been reported by Jardeleza et al. ${ }^{23}$ this is considered due to a nutritional role of ISMN at sub-antimicrobial levels.

MIC and MBEC assay of ISMN loaded PLGA-MP. Owing to the requirement for high ISMN concentrations to exert antibacterial activity, PLGA nanoparticles were not considered for further antimicrobial evaluation due to their low drug loads. This section therefore focuses of ISMN containing PLGA microparticles (ISMN-PLGA-MP). The apparent MIC for ISMN encapsulated in PLGA-MPs was determined as $7.5 \mathrm{mg} \mathrm{mL}^{-1}$ (Table 5). The MIC observed for encapsulated ISMN showed a two-fold increase compared to that of the free ISMN $\left(3.75 \mathrm{mg} \mathrm{mL}^{-1}\right)$. The MBEC concentration for free ISMN was $15 \mathrm{mg} \mathrm{mL}^{-1}$, which is
Table 5 MIC and MBEC values for ISMN formulations

\begin{tabular}{llc}
\hline & MIC $\left(\mathrm{mg} \mathrm{mL}^{-1}\right)$ & MBEC $\left(\mathrm{mg} \mathrm{mL}^{-1}\right)$ \\
\hline Free ISMN & 3.75 & 15 \\
MP-encapsulated ISMN & 7.5 & 30
\end{tabular}

in accordance with previous studies, ${ }^{23}$ whereas the measured MBEC concentration for encapsulated ISMN in PLGA-MPs was determined as $30 \mathrm{mg} \mathrm{mL}^{-1}$ (Table 5). The higher MBEC values relative to MIC observed for both free and encapsulated ISMN are likely due to the incomplete drug release from PLGA-MPs within $24 \mathrm{~h}$ of incubation, effectively reducing the available drug concentration compared to treatment with a simple ISMN solution. An increase in the apparent MIC and MBEC of sustained release PLGA particles relative to drug solution has previously been observed for the antibiotic ciprofloxacin. ${ }^{39}$

Anti-planktonic and biofilm effects of ISMN loaded PLGA-MP. The influence of a 1 hour ISMN treatment (at both 10 and $60 \mathrm{mg} \mathrm{mL}^{-1}$ ) dosed as either ISMN solutions or PLGA MP's on $S$. aureus in planktonic cultures grown for 24 hours are shown in Fig. 8. Free ISMN at both 60 and $10 \mathrm{mg} \mathrm{mL}{ }^{-1}$ significantly decreased $(P<0.05)$ the number of viable bacteria, i.e. a $\sim 86 \%$ and $\sim 30 \%$ reduction respectively. ISMN-PLGA-MP inhibited bacterial growth with less effectiveness than the free ISMN and this was only significant at $60 \mathrm{mg} \mathrm{mL}^{-1}$ ISMN. The blank PLGAMPs exhibited no statistically significant inhibition of bacterial growth, highlighting that the antibacterial effects were elicited by ISMN itself. The anti-biofilm effects of ISMN were investigated in vitro using $S$. aureus biofilms grown in 96-well plates as previously described; these were challenged with both free ISMN and ISMN-loaded PLGA-MPs for 24 hours. As illustrated in Fig. 9, the percentage of bacterial cell viability following exposure to free ISMN (60 $\mathrm{mg} \mathrm{mL}^{-1}$ ) was significantly reduced (57.8\%, $p>0.05)$, whereas enhanced biofilm formation was observed for free ISMN at $10 \mathrm{mg} \mathrm{mL}^{-1}$ concentration. In agreement with previous observations, a low concentration of ISMN was associated with a stimulatory biofilm effect. PLGA-MPs containing $60 \mathrm{mg} \mathrm{mL} \mathrm{m}^{-1}$ ISMN exhibited a statistically significant anti-biofilm effect (42.2\% bacterial viability reduction, $p<0.05)$, but with less potency than free ISMN ( $57.8 \%$ bacterial viability reduction).
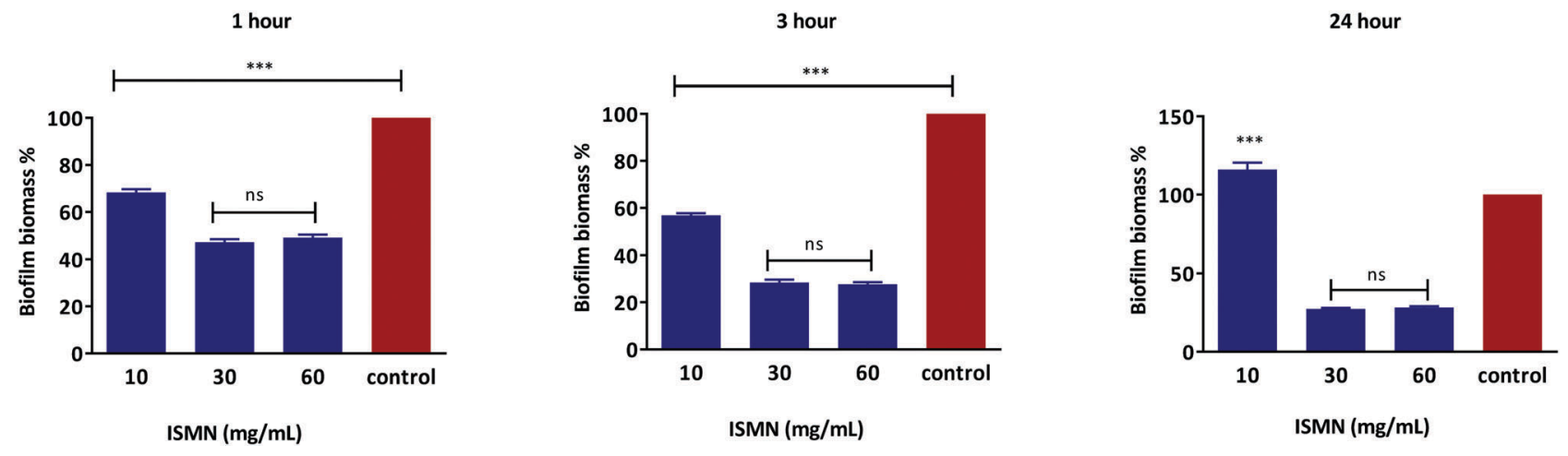

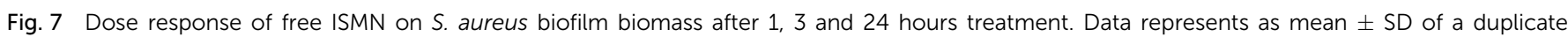
experiment. 


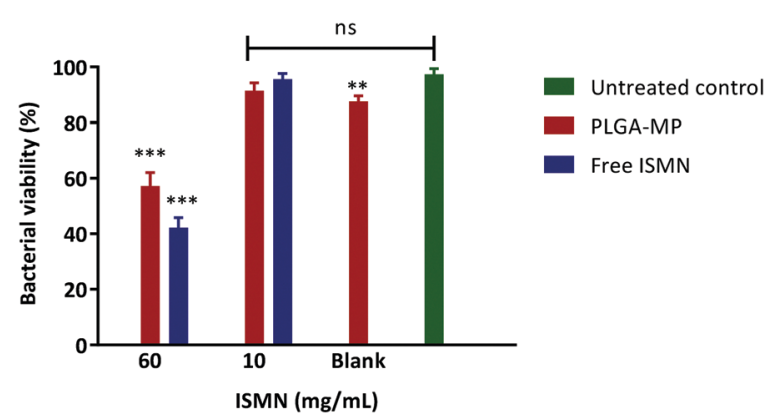

Fig. 8 Percentage of viable bacterial cells after $1 \mathrm{~h}$ treatments on planktonic S. aureus bacteria. PLGA MP-60 and 10: PLGA MP loaded with 60 and $10 \mathrm{mg} \mathrm{mL}^{-1}$ of ISMN and ISMN-60 and 10: free ISMN with 60 and $10 \mathrm{mg} \mathrm{mL}^{-1}$. Data represents as mean \pm SD of a duplicate experiment.

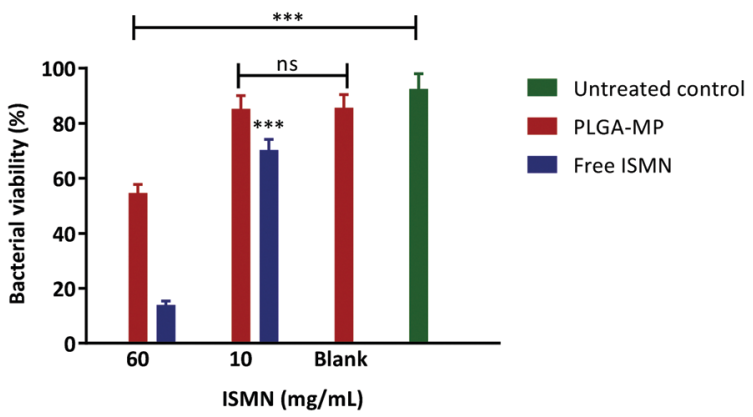

Fig. 9 Percentage of viable bacterial cells after $24 \mathrm{~h}$ treatments on S. aureus biofilm. Data represents as mean \pm SD of a duplicate experiment.

The anti-biofilm effect of blank microparticles and $10 \mathrm{mg} \mathrm{mL}^{-1}$ ISMN encapsulated in PLGA-MPs resulted in no statistically significant reduction of biofilm viability compared to the untreated control $(p>0.05)$. Subsequent analysis of ISMN release from PLGA-MPs demonstrated that the initial burst release followed by a constant sustained release enhanced the diffusion of ISMN from PLGA-MPs into biofilm. Therefore, continuous exposure of the biofilm to ISMN through sustained release from PLGA-MPs enhanced the efficacy compared to free ISMN despite incomplete ISMN release after 24 hours. Given the difficulty of biofilm penetration by PLGA-MPs, it may be possible to improve efficacy by modifying microparticle surfaces with a cationic polymer such as chitosan, potentially increasing the interaction with anionic mucopolysaccharide in the biofilm and maximizing biofilm exposure to ISMN. This is an area for future investigation.

\section{Conclusion}

A double emulsion solvent evaporation method has been successfully optimised to encapsulate the NO donor ISMN into PLGA nanoparticles and microparticles. Considerable sustained release of ISMN has been demonstrated. PLGA microparticles but not nanoparticles were able to deliver sufficient levels of ISMN to planktonic and biofilms of $S$. aureus to cause significant antibacterial effects. ISMN-PLGA-MP formulations have potential for the treatment of a number of biofilm-based infections.

\section{Acknowledgements}

This project has been supported by The National Health and Medical Research Council (NHMRC project grant GNT1047576 and GNT1090898). The University of South Australia and School of Pharmacy and Medical Sciences are acknowledged for providing a scholarship to SH. This work was performed in part at the South Australian node of the Australian National Fabrication Facility (ANFF) under the National Collaborative Research Infrastructure Strategy to provide nano- and microfabrication facilities for Australia's researchers.

\section{References}

1 G. A. James, E. Swogger, R. Wolcott, E. D. Pulcini, P. Secor, J. Sestrich, J. W. Costerton and P. S. Stewart, Wound Repair Regen., 2008, 16, 37-44.

2 R. A. Brady, J. G. Leid, J. H. Calhoun, J. W. Costerton and M. E. Shirtliff, FEMS Immunol. Med. Microbiol., 2008, 52, 13-22.

3 B. A. Cunha, M. V. Gill and J. M. Lazar, Infect. Dis. Clin. North Am., 1996, 10, 811-834.

4 A. Foreman and P.-J. Wormald, Laryngoscope, 2010, 120, 1701-1706.

5 R. O. Darouiche, N. Engl. J. Med., 2004, 350, 1422-1429.

6 G. E. Thwaites, J. D. Edgeworth, E. Gkrania-Klotsas, A. Kirby, R. Tilley, M. E. Török, S. Walker, H. F. L. Wertheim, P. Wilson and M. J. Llewelyn, Lancet Infect. Dis., 2011, 11, 208-222.

7 P. M. Hawkey, J. Antimicrob. Chemother., 2008, 62, i1-i9.

8 B. Vu, M. Chen, R. Crawford and E. Ivanova, Molecules, 2009, 14, 2535.

9 N. Høiby, T. Bjarnsholt, M. Givskov, S. Molin and O. Ciofu, Int. J. Antimicrob. Agents, 2010, 35, 322-332.

10 I. d'Angelo, C. Conte, M. I. La Rotonda, A. Miro, F. Quaglia and F. Ungaro, Adv. Drug Delivery Rev., 2014, 75, 92-111.

11 T.-F. C. Mah and G. A. O’Toole, Trends Microbiol., 2001, 9, 34-39.

12 C. A. Fux, J. W. Costerton, P. S. Stewart and P. Stoodley, Trends Microbiol., 2005, 13, 34-40.

13 P. S. Stewart, J. Bacteriol., 2003, 185, 1485-1491.

14 N. Høiby, O. Ciofu and T. Bjarnsholt, Future Microbiol., 2010, 5, 1663-1674.

15 J. W. Costerton, P. S. Stewart and E. P. Greenberg, Science, 1999, 284, 1318-1322.

16 C. A. Gordon, N. A. Hodges and C. Marriott, J. Antimicrob. Chemother., 1988, 22, 667-674.

17 G. N. Marcells, P. S. Phillips, N. A. Cohen, R. J. Harvey and R. Sacks, Otolaryngol.-Head Neck Surg., 2011, 144, 159-169.

18 D. A. Sanchez, J. Nosanchuk and A. Friedman, Nanomedicine, 2012, 7, 933-936.

19 J. Saraiva, S. S. Marotta-Oliveira, S. A. Cicillini, J. D. O. Eloy and J. M. Marchetti, J. Drug Delivery, 2011, 936438.

20 P. G. Wang, M. Xian, X. Tang, X. Wu, Z. Wen, T. Cai and A. J. Janczuk, Chem. Rev., 2002, 102, 1091-1134.

21 A. Friedman and J. Friedman, Expert Opin. Drug Delivery, 2009, 6, 1113-1122. 
22 C. Jardeleza, A. Foreman, L. Baker, S. Paramasivan, J. Field, L. W. Tan and P. J. Wormald, International Forum of Allergy and Rhinology, 2011, 1, 438-444.

23 C. Jardeleza, S. Rao, B. Thierry, P. Gajjar, S. Vreugde, C. A. Prestidge and P. J. Wormald, PLoS One, 2014, 9, e92117.

24 E. M. Hetrick, J. H. Shin, H. S. Paul and M. H. Schoenfisch, Biomaterials, 2009, 30, 2782-2789.

25 E. M. Hetrick, J. H. Shin and M. H. Schoenfisch, 8th World Biomaterials Congress 2008, 2008, 4, 1845.

26 A. J. Friedman, G. Han, M. S. Navati, M. Chacko, L. Gunther, A. Alfieri and J. M. Friedman, Nitric Oxide, 2008, 19, 12-20.

27 L. R. Martinez, G. Han, M. Chacko, M. R. Mihu, M. Jacobson, P. Gialanella, A. J. Friedman, J. D. Nosanchuk and J. M. Friedman, J. Invest. Dermatol., 2009, 129, 2463-2469.

28 J. Panyam and V. Labhasetwar, Adv. Drug Delivery Rev., 2003, 55, 329-347.

29 R. Jain, N. H. Shah, A. W. Malick and C. T. Rhodes, Drug Dev. Ind. Pharm., 1998, 24, 703-727.

30 H. K. Makadia and S. J. Siegel, Polymers, 2011, 3, 1377-1397.

31 K. Dillen, J. Vandervoort, G. Van den Mooter, L. Verheyden and A. Ludwig, Int. J. Pharm., 2004, 275, 171-187.

32 J. Liu, Z. Qiu, S. Wang, L. Zhou and S. Zhang, Biomed. Mater., 2010, 5, 065002.

33 J. O'Sullivan, M. Park and J. Beevers, J. Cereal Sci., 2016, 69, 77-84.

34 C. A. Schneider, W. S. Rasband and K. W. Eliceiri, Nat. Methods, 2012, 9, 671-675.

35 I. Wiegand, K. Hilpert and R. E. W. Hancock, Nat. Protoc., 2008, 3, 163-175.

36 J. H. Merritt, D. E. Kadouri and G. A. O'Toole, Current Protocols in Microbiology, 2011.

37 E. Peeters, H. J. Nelis and T. Coenye, J. Microbiol. Methods, 2008, 72, 157-165.
38 I. Vandecandelaere, H. Van Acker and T. Coenye, Methods Mol. Biol., 2016, 1333, 53-66.

39 N. Thomas, C. Thorn, K. Richter, B. Thierry and C. Prestidge, J. Pharm. Sci., 2016, 105, 3115-3122.

40 U. Bilati, E. Allémann and E. Doelker, Pharm. Dev. Technol., 2003, 8, 1 .

41 U. Bilati, E. Allémann and E. Doelker, J. Microencapsulation, 2005, 22, 205-214.

42 T. Verrecchia, G. Spenlehauer, D. V. Bazile, A. Murry-Brelier, Y. Archimbaud and M. Veillard, J. Controlled Release, 1995, 36, 49-61.

43 A. Rafati, A. Boussahel, K. M. Shakesheff, A. G. Shard, C. J. Roberts, X. Chen, D. J. Scurr, S. Rigby-Singleton, P. Whiteside, M. R. Alexander and M. C. Davies, J. Controlled Release, 2012, 162, 321-329.

44 J. G. Y. Chan, H. K. Chan, C. A. Prestidge, J. A. Denman, P. M. Young and D. Traini, Eur. J. Pharm. Biopharm., 2013, 83, 285-292.

45 A. M. Belu, M. C. Davies, J. M. Newton and N. Patel, Anal. Chem., 2000, 72, 5625-5638.

46 N. Faisant, J. Siepmann, J. Richard and J. P. Benoit, Eur. J. Pharm. Biopharm., 2003, 56, 271-279.

47 A. N. Ford Versypt, D. W. Pack and R. D. Braatz, J. Controlled Release, 2013, 165, 29-37.

48 R. W. Korsmeyer, R. Gurny, E. Doelker, P. Buri and N. A. Peppas, Int. J. Pharm., 1983, 15, 25-35.

49 J. Vysloužil, P. Doležel, M. Kejdušová, V. Košál, L. Beneš and K. Dvoráčková, Pharm. Dev. Technol., 2016, 21, 214-221.

50 S. R. Jameela, N. Suma and A. Jayakrishnan, J. Biomater. Sci., Polym. Ed., 1997, 8, 457-466.

51 C. Ahlneck and G. Zografi, Int. J. Pharm., 1990, 62, 87-95.

52 F. Cui, D. Cun, A. Tao, M. Yang, K. Shi, M. Zhao and Y. Guan, J. Controlled Release, 2005, 107, 310-319.

53 H. K. Makadia and S. J. Siegel, Polymers, 2011, 3, 1377-1397. 